Ann. Génét. Sél. anim., r974, 6 (4), 463-476.

\title{
EFFETS DE DIFFÉRENTES FRÉQUENCES DE TRAITE SUR LA PRODUCTION LAITIERE DES CAPRINS
}

\author{
J. C. MOCQUOT et T. AURAN (1) \\ avec la collaboration technique de P. Gurllimin et D. TANGly \\ Station de Génétique quantitative et appliquée, \\ Centre national de Recherches zootechniques, I. N. R. A., \\ 78350 Jouy en Josas
}

\section{RÉSUMÉ}

La réduction du nombre de traites des femelles laitières peut contribuer à l'amélioration de leurs conditions d'exploitation, dans la mesure où la production laitière ne serait pas réduite dans des proportions trop importantes. La sélection d'animaux adaptés à des conditions variées de traite peut être envisagée à moindres frais pour les caprins. Aussi avons-nous analysé ici les répercussions phénotypiques de différents systèmes de traite sur la production laitière des chevrettes en première lactation. 83 femelles croisées de type alpin (Chamoisée $\times$ Saanen) nées en 1972 dans notre troupeau expérimental ont été réparties en trois lots avec les traitements suivants : lot IX : une traite par jour; lot $2 \mathrm{X}: 2$ traites par jour; lot $2 \mathrm{X}$. IX : deux traites pendant deux mois puis une seule traite.

La différence de production entre les lots $\mathrm{IX}$ et $2 \mathrm{X}$ est maximum, et de l'ordre de $50 \mathrm{p}$. Ico, au voisinage du deuxième mois de lactation, inférieure ensuite du fait d'une meilleure persistance des productions en une seule traite. Globalement et par rapport au lot $2 \mathrm{X}$ les différences de production totale sont respectivement de $20 \mathrm{p}$. Ioo et $45 \mathrm{p}$. Ioo pour les lots $2 \mathrm{X}$. I X et IX. Les taux butyreux et azoté sont, quant à eux, légèrement supérieurs pendant les $2 / 3$ de la lactation pour le lot $\mathrm{IX}$ mais restent comparables à ceux du lot $2 \mathrm{X}$ pour le lot $2 \mathrm{X}$. I X malgré le passage à une traite en cours de lactation. La suppression d'une traite pendant tout ou partie de la lactation tend à provoquer en moyenne un tarissement plus précoce $(-\mathrm{I} 4 \mathrm{j}$ pour le lot $\mathrm{IX}$ et $--\mathrm{I} 2 \mathrm{j}$ pour le lot $2 \mathrm{X}$. IX), elle tend également, du fait des quantités produites plus faibles, à accroître les liaisons positives et à réduire les oppositions phénotypiques entre la quantité de lait et les taux. La variabilité des performances enregistrées avec une seule traite par jour est supérieure en terme de coefficient de variation, notamment en ce qui concerne la persistance pour le lot $2 \mathrm{X}$. IX. Ceci semble traduire des différences importantes d'adaptation individuelle, différences confirmées par une distribution non normale des productions obtenues avec une seule traite. Enfin si on n'observe pas de différences entre traitements quant à l'état sanitaire de la mamelle, la suppression d'une traite entraîne une élévation importante du nombre des leucocytes du lait.

En conclusion la sélection d'animaux adaptés à une seule traite risque d'être efficace, au moins à court terme, mais ne se justifie que si elle permet d'obtenir des aninaux également mieux adaptés à des conditions variées de traite simplifiée.

(1) Adresse permanente : Institut de Génétique animale, Université agricole de Norvège, AS-NLH (Norvège). 


\section{INTRODUC'TION}

La réduction des contraintes humaines liées à la traite des femelles laitières constitue depuis longtemps une préoccupation importante des chercheurs zootechniciens. On peut envisager de réduire le temps consacré à la traite de ces femelles soit en augmentant le nombre d'animaux traits par heure, par exemple en sélectionnant sur le temps de traite, soit en réduisant le nombre de traites pratiquées par animal et par lactation. Cette dernière possibilité a conduit, vers les années r940, à réduire effectivement pour les bovins de 3 à 2 le nombre de traites journalières. On peut envisager d'aller au-delà encore, et plusieurs auteurs ont expérimenté la pratique d'une seule traite par jour pendant tout ou partie de la lactation sur les bovins : WoodwARD (I93I), Clatesson et al. (I959), LABussiere (I968) ; ou sur les ovins : Morag (I968), CASU et LABUSSIERE (I972). Aucune expérience ne semble avoir été réalisée avec des chèvres et, pour les bovins au moins, les répercussions phénotypiques sur la production laitière sont telles que ces essais n'ont pu mettre en évidence de solution satisfaisante sur le plan économique. La sélection d'animaux adaptés à une seule traite est indispensable si cette simplification extrême des opérations de traite doit être envisagée. Elle peut également s'avérer intéressante dans des conditions moins sévères, par exemple dans le cas de suppression d'une ou deux traites hebdomadaires, dans la mesure où la pratique d'une seule traite permettrait de mieux discréminer les reproducteurs pour leurs aptitudes génétiques d'adaptation et de production dans des conditions d'exploitation variées. Une telle sélection expérimentale n'a pas été envisagée, à notre connaissance, en raison des pertes élevées qu'elle entrainerait pour des bovins. Dans la mesure où il existe une analogie certaine entre les paramètres de production laitière des bovins et des caprins, on peut penser qu'un essai conduit sur des chèvres permettrait d'obtenir à moindre frais une réponse plus rapide sur l'éventuel intérêt d'une application à grande échelle. Pour ces différentes raisons, il nous a semblé utile d'analyser les répercussions phénotypiques de quelques-unes de ces techniques simplifiées de traite sur la production laitière des chèvres.

\section{MATÉRIEL ET MÉTHODES}

Les données utilisées ont été recueillies en 1973 dans le troupeau expérimental de l'I. N. R A. situé au Domaine de Galle à Avord (Cher). Elles correspondent aux premières lactations des 83 chevrettes nées et élevées dans ce troupeau en 1972 , toutes issues de I I boucs de race Alpine Chamoisée, 39 d'entre elles étant de mère de race Alpine Saanen et 44 de mères croisées : Saanen $\times$ Chamoisée.

\section{Conduite du troupeau}

Les animaux sont maintenus toute l'année à l'intérieur en stabulation libre, avec affouragement en vert de mai à septembre (luzerne, trèfle incarnat, vesce avoine, maïs, sorgho) et distribution de foin (dactyle luzerne) et de luzerne déshydratée. Ils disposent par ailleurs, à volonté, d'un mélange de céréales, sous forme granulée et reçoivent environ $500 \mathrm{~g}$ de concentré lors de la traite, quantité en générale non consommée dans sa totalité. 
La traite est effectuée mécaniquement, sans préparation de la mamelle et avec un très léger égouttage machine pour certains animaux, dans une installation "Herringbone " de Io postes sur Lactoduc ligne basse, avec un rapport de pulsations de $3 / 1$ pour une vitesse de $60 / \mathrm{mn}$.

Tous les animaux encore en lactation ont été taris le I 8 décembre 1973.

\section{Constitution des lots expérimentaux}

Les 83 chevrettes ont mis bas entre le I 5 février et le 30 avril r973. Elles ont été soumises, dès la mise bas, à deux traites journalières pendant les 5 premiers jours de lactation puis réparties en trois lots homogènes vis-à-vis : de la date de mise bas, du génotype, de l'ascendance paternelle et de la production laitière initiale. Elles ont été soumises aux traitements suivants durant leur première lactation.

Lot IX : 27 chevrettes. Une traite par jour effectuée le matin.

Lot $2 \mathrm{X}$ : 28 chevrettes. Deux traites par jour (matin et soir) espacées de io heures 40 minutes \pm I heure I 2 minutes.

Lot 2 X. IX : 28 chevrettes. Deux traites par jour en début de lactation une traite le matin ensuite (Le changement de système étant effectif le lendemain du quatrième contrôle laitier : cinquante quatrième jour $\pm 4,8$ jours).

\section{Contrôles expérimentaux}

Les contrôles de production laitière quantitative ont été effectués par pesée tous les $\mathrm{I}_{4}$ jours. Les taux butyreux et azoté ont été déterminés par analyse automatique (méthode Gerber pour la matière grasse et au Noir Amido pour la matière azotée) d'un échantillon de $30 \mathrm{ml}$ de lait (proportionnel dans le cas de deux traites) prélevé tous les I $_{4}$ jours sur la moitié des lots, c'est-à-dire une fois par mois pour chaque chèvre.

L'état sanitaire de la mamelle a été vérifié à l'aide d'un Californian Mastitis Test (CMT) mensuel dont les résultats ont été appréciés par quatre classes de réaction $(o,+,++$ et +++$)$, et de trois comptages des leucocytes du lait réalisés par la méthode " coulter counter"au Laboratoire de la Station expérimentale laitière à Poligny (Jura) sur des échantillons prélevés à date fixe : 3 mai, I 8 juin et Io août.

Le poids vif des animaux a été apprécié par pesées simples réalisées dans les 48 heures après la mise bas de chaque chevrette, puis ensuite à date fixe, les $17-07$ et 22-10 ainsi que lors du changement de système au quatrième contrôle pour le lot $2 \mathrm{X}$. IX.

\section{Caractères étudiés}

Outre les productions totales de lait, de matière grasse et de matière azotée par lactation nous avons calculé pour chaque chevrette les productions partielles cumulées de la mise bas au quatrième contrôle, du quatrième au huitième contrôle. De ce fait le coefficient de persistance a été simplement estimé par le rapport de la production après le huitième contrôle à la production entre le quatrième et le huitième contrôle, estimation qui prend en compte à la fois la décroissance de production laitière après le quatrième contrôle et la durée de lactation.

\section{RÉSULTATS}

Quantité de lait.

\section{I. - Courbes de lactation}

L'évolution des productions moyennes par numéro de contrôle est représentée sur la figure I pour chaque lot. Nous y avons également fait figurer l'évolution de la production moyenne du lot $2 \mathrm{X}$ à la traite du matin. L'examen de ces courbes de lactation permet de constater un maximum de production au voisinage du cinquième contrôle $(\simeq 2$ mois) pour les animaux soumis à deux traites, un maximum plus tardif 
et très peu marqué pour le lot $\mathrm{IX}$. Ce lot expérimental présente une courbe de lactation voisine de celle obtenue à la traite du matin avec le lot témoin $2 \mathrm{X}$, avec cependant une production maximum moins prononcée mais une persistance supérieure. D'une manière générale ces courbes traduisent une persistance des lactations d'autant moins bonne que la production est plus élevée, ce que confirme l'examen des coefficients moyens de persistance présentés au tableau $\mathrm{I}$. De ce fait les écarts de production entre les différents traitements sont maximum au voisinage du cinquième contrôle, où ils atteignent 50 p. IOO, et tendent à s'atténuer par la suite.

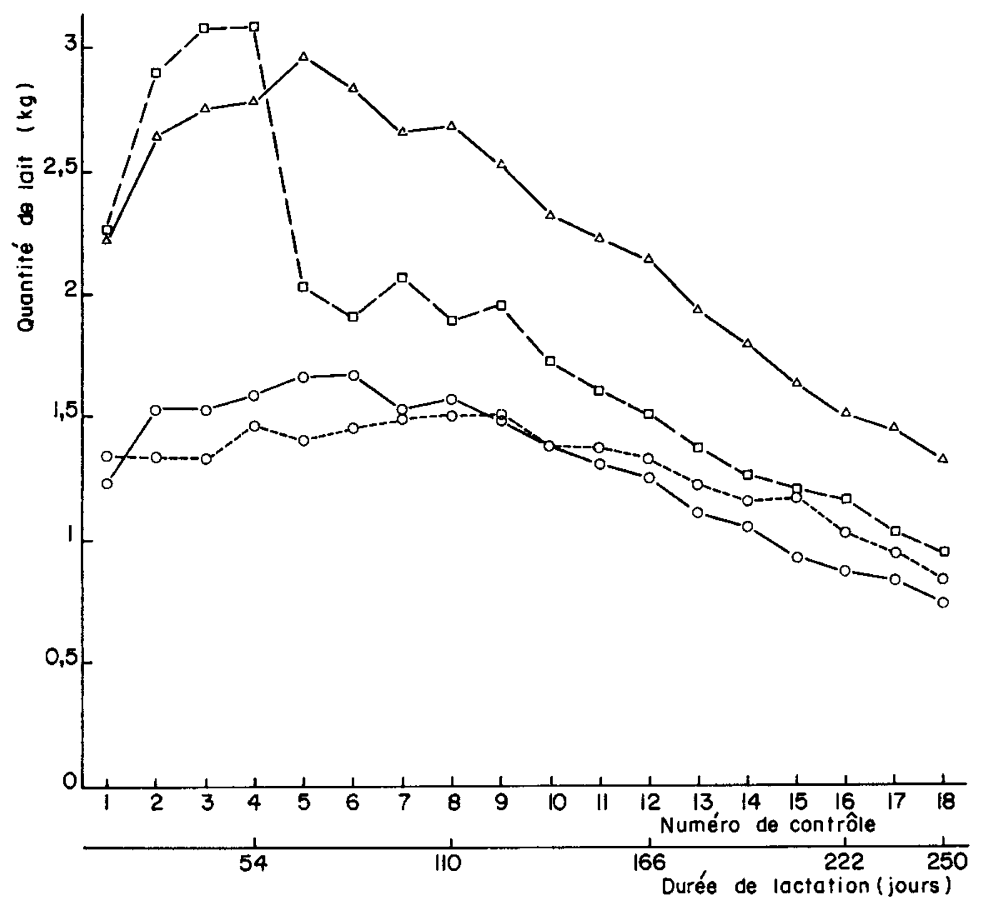

Fı́. I. - Évolution de la quantité movenne de lait produite aut cours de la lactation o-..-O Lot IX

ㄴ-口 Lot $2 X$. IX

$\triangle \longrightarrow$ Lot $2 \mathrm{X}$

0 Lot $2 \mathrm{X}$ à la traite du matin

TABLEAU I

Moyennes et variances des coefficients de persistance

\begin{tabular}{c|c|c}
\hline Lot expérimental & Noyenne & Variance \\
\hline $1 \times$ & 2,18 & 0,374 \\
\hline $2 \times 1 \times$ & 2,04 & 0,679 \\
\hline $2 \times$ & 1,85 & 0,204 \\
\hline
\end{tabular}




\section{Constituants du lait.}

Le contrôle des taux ayant lieu pour chaque chevrette une fois par mois seulement, chaque lot expérimental est en fait constitué de deux échantillons : animaux prélevés lors des contrôles laitiers d'ordre impair et animaux prélevés lors des contrôles d'ordre pair. Du fait du hasard ces échantillons présentaient en général pour un même traitement des différences importantes de moyennes des taux butyreux et azotés pondérés avec cependant la même évolution au cours de la lactation. Pour la représenter nous avons reporté sur la figure 2 , pour chaque lot, la moyenne des deux échantillons prélevés lors de contrôles consécutifs.
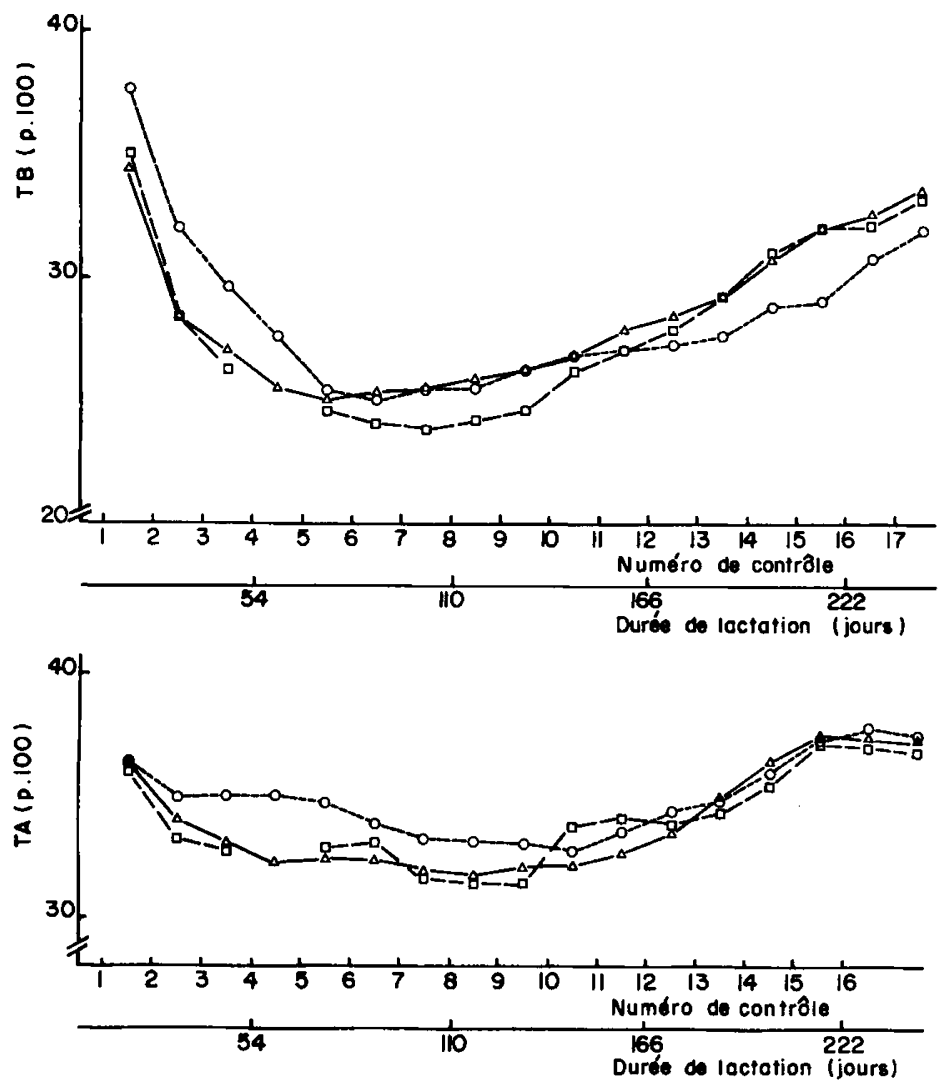

FrG. 2. - Evolution des taux butyreux et azote movens pendant la lactation o--.- O Lot IX

$\square--\square$ Lot $2 X$. IX

Ces courbes font apparaître pour chacun des lots l'évolution habituelle des taux au cours de la lactation, évolution plus accentuée pour le taux butyreux que pour le taux azoté. Globalement on constate des taux décroissants légèrement plus élevés en début de lactation pour les animaux soumis à une seule traite, taux qui s'accroissent ensuite d'autant plus vite que la persistance moyenne des lactations correspondantes 
est plus faible. Ainsi, le taux butyreux du lot $2 \mathrm{X}$ est équivalent à celui du lot $\mathrm{IX}$ dès le sixième contrôle et supérieur en fin de lactation. La même valeur moyenne n'est atteinte qu'en fin de lactation pour le taux azoté. Les taux du lot $2 X$. IX restentinférieurs à ceux du lot $\mathrm{rX}$, malgré le passage à une seule traite, pour n'atteindre des valeurs équivalentes, voire supérieures, qu'en fin de lactation lors de la décroissance importante des quantités de lait produites.

\section{Quantités et taux.}

\section{2. - Productions totales}

Les moyennes, écart types et coefficients de variation des productions cumulées de lait, de matière grasse et de matière azotée pendant les différentes phases de la lactation sont présentés au tableau 2 ; les paramètres de distribution des taux buty-

\section{TABLEAU 2} Effet de différentes fréquences de traite sur les productions partielles et totales
en première lactation

$(\mathrm{X}=$ moyenne $; \quad \sigma=$ écart-type $\quad \mathrm{CV}=$ coefficient de variation $)$

\begin{tabular}{|c|c|c|c|c|}
\hline & & \multicolumn{3}{|c|}{ Lots expérimentaux } \\
\hline & & $1 \mathrm{X}$ & $2 \mathrm{X} .1 \mathrm{X}$ & $2 \mathrm{X}$ \\
\hline \multicolumn{2}{|c|}{ Productions cumulées de lait $(\mathrm{kg})$} & & & \\
\hline $\begin{array}{c}\text { De la mise bas } \\
\text { au quatrième contrôle } \\
\text { (54 jours) }\end{array}$ & $\begin{array}{c}\bar{x} \\
\sigma \\
C V\end{array}$ & $\begin{array}{l}72,4 \\
27,8 \\
0,384\end{array}$ & $\begin{array}{l}155,7 \\
38,7 \\
0,2 \div 9\end{array}$ & $\begin{array}{l}141,6 \\
50,3 \\
0,355\end{array}$ \\
\hline $\begin{array}{l}\text { Du quatrième } \\
\text { au huitième contrôle } \\
\text { ( } 56 \text { jours })\end{array}$ & $\begin{array}{c}\bar{x} \\
\sigma \\
C V\end{array}$ & $\begin{array}{l}81,1 \\
31,4 \\
0,388\end{array}$ & $\begin{array}{l}109,3 \\
36,2 \\
0,332\end{array}$ & $\begin{array}{l}157,6 \\
39,4 \\
0,250\end{array}$ \\
\hline Lactation totale & $\begin{array}{c}\bar{x} \\
\sigma \\
c V\end{array}$ & $\begin{array}{l}323,7 \\
130,2 \\
0,402\end{array}$ & $\begin{array}{l}479,6 \\
145,7 \\
\quad 0,250\end{array}$ & $\begin{array}{l}589,6 \\
120,0 \\
0,247\end{array}$ \\
\hline \multicolumn{5}{|c|}{ Productions totalcs (kg) } \\
\hline Matière grasse & $\begin{array}{c}\overline{\mathrm{X}} \\
\sigma \\
\mathrm{CV}\end{array}$ & $\begin{array}{l}9,5 \\
3,4 \\
0,358\end{array}$ & $\begin{array}{l}13,3 \\
3,24 \\
0,244\end{array}$ & $\begin{array}{l}16,6 \\
4,27 \\
0,257\end{array}$ \\
\hline Matière azotée & $\begin{array}{c}\overline{\mathrm{X}} \\
\sigma \\
\mathrm{CV}\end{array}$ & $\begin{array}{l}11,6 \\
4,13 \\
0,356\end{array}$ & $\begin{array}{l}16,1 \\
4,3 \\
0,267\end{array}$ & $\begin{array}{l}20,0 \\
5,3 \\
0,265\end{array}$ \\
\hline
\end{tabular}

reux et azotés correspondants au tableau 3. Ces chiffres mettent en évidence une production initiale supérieure du lot $2 \mathrm{X}$. IX par rapport au lot $2 \mathrm{X}$ pris comme témoin, alors que les animaux étaient conduits ensembles durant cette période. 
Compte tenu de cette différence, vraisemblablement liée aux erreurs d'échantillonnage, il semble que la production des animaux du lot $2 \mathrm{X}$. IX après le passage à une traite demeure supérieure à celle du lot IX pendant la même période et que l'on puisse estimer globalement à $20 \mathrm{p}$. Ioo la différence de production totale de lait du lot $2 \mathrm{X}$. IX et à $45 \mathrm{p}$. Ioo celle du lot IX par rapport à la production témoin du lot $2 \mathrm{X}$.

TABLEAU 3

Effet de différentes fréquences de traite sur les taux butyreux et azotés moyens

\begin{tabular}{|c|c|c|c|c|}
\hline \multirow{2}{*}{$\begin{array}{c}\text { Période } \\
\text { de la lactation }\end{array}$} & \multirow{2}{*}{ Variables } & \multicolumn{3}{|c|}{ Lots expérimentaux } \\
\hline & & $1 \mathrm{X}$ & $2 \mathrm{X} .1 \mathrm{X}$ & $2 \mathrm{X}$ \\
\hline \multirow{2}{*}{$\begin{array}{c}\text { De la mise bas } \\
\text { au quatrième contrôle } \\
\left(5^{4} \text { jours }\right)\end{array}$} & $\mathrm{TB}\left\{\begin{array}{l}\overline{\mathrm{X}} \\
\mathrm{CV}\end{array}\right.$ & $\begin{array}{l}3,46 \\
0,219\end{array}$ & $\begin{array}{l}3,14 \\
0,141\end{array}$ & $\begin{array}{l}3,10 \\
0,155\end{array}$ \\
\hline & $\mathrm{TA}\left\{\begin{array}{l}\overline{\mathrm{X}} \\
\mathrm{CV}\end{array}\right.$ & $\begin{array}{l}3,58 \\
0,074\end{array}$ & $\begin{array}{l}3,43 \\
0,063\end{array}$ & $\begin{array}{l}3,50 \\
0,077\end{array}$ \\
\hline \multirow{2}{*}{$\begin{array}{c}\text { Du quatrième } \\
\text { au huitième contrôle } \\
\text { ( } 56 \text { jours) }\end{array}$} & $\mathrm{TB}\left\{\begin{array}{l}\overline{\mathrm{X}} \\
\mathrm{CV}\end{array}\right.$ & $\begin{array}{l}2,54 \\
0,150\end{array}$ & $\begin{array}{l}2,42 \\
0,144\end{array}$ & $\begin{array}{l}2,51 \\
0,130\end{array}$ \\
\hline & TA $\left\{\begin{array}{l}\bar{X} \\
C V\end{array}\right.$ & $\begin{array}{l}3,43 \\
0,072\end{array}$ & $\begin{array}{l}3,20 \\
0,079\end{array}$ & $\begin{array}{l}3,22 \\
0,075\end{array}$ \\
\hline \multirow{2}{*}{ Lactation totale } & TB $\left\{\begin{array}{l}\bar{x} \\
\mathrm{CV}\end{array}\right.$ & $\begin{array}{l}2,86 \\
0,126\end{array}$ & $\begin{array}{l}2,81 \\
0,109\end{array}$ & $\begin{array}{l}2,84 \\
0,116\end{array}$ \\
\hline & $\mathrm{TA}\left\{\begin{array}{l}\overline{\mathrm{X}} \\
\mathrm{CV}\end{array}\right.$ & $\begin{array}{l}3,47 \\
0,069\end{array}$ & $\begin{array}{l}3,36 \\
0,062\end{array}$ & $\begin{array}{l}3,37 \\
0,062\end{array}$ \\
\hline
\end{tabular}

Les taux pondérés moyens des animaux soumis à une seule traite au long de la lactation sont supérieurs, nous l'avons vu, au moins pendant la moitié de la lactation ; les taux moyens du lot $2 \mathrm{X}$. IX après passage à une traite restent, quant à eux, comparables à ceux observés avec deux traites par jour. De ce fait les différences de productions totales de matière grasse et de matière azotée, par rapport au lot $2 \mathrm{X}$, sont plutôt supérieures pour le lot $2 \mathrm{X}$. IX et inférieures pour le lot IX à celles observées pour la quantité de lait.

\section{Durée de lactation.}

Nous avons présenté au tableau 4 les conséquences des différents traitements sur la durée de lactation en tenant compte du tarissement provoqué chez certaines chevrettes.

La suppression d'une traite pendant tout ou partie de la lactation tend à provoquer un tarissement en moyenne plus précoce, la majorité des lactations ayant cependant une durée supérieure à 250 jours. 


\section{TABLEAU 4}

Effet de différentes fréquences de traite sur la durée de lactation des chevrettes

\begin{tabular}{c|c|c|c}
\hline \hline & Lot 1X & Lot 2X. 1X & Lot 2X \\
& & & \\
\hline Nombre de tarissements : & & & \\
- naturels & 20 & 16 & 10 \\
- provoqués & 7 & 12 & 18 \\
\hline Durée moyenne & 258 jours & 256 jours & 270 jours \\
de lactation & & & \\
\hline Nombre de lactations : & & 2 & 0 \\
$<200$ jours & 2 & 6 & 2 \\
$<250$ jours & 8 & 26 & 1 \\
$<300$ jours & 0 & 2 & 27 \\
$>300$ jours & & & \\
\hline
\end{tabular}

\section{3. - Liaisons phénotypiques entre variables}

Les coefficients de corrélation phénotypiques observés, pour chaque lot, entre les différents critères de production en i ro jours de lactation figurent au tableau 5 .

\section{TABLEAU 5}

Effet de différentes fréquences de traite sur les liaisons phénotypiques observées entre caractèves de production laitière en 110 jours de lactation

\begin{tabular}{|c|c|c|c|c|c|}
\hline \multicolumn{2}{|c|}{ Variables } & Quantité & Quantité & $\begin{array}{c}\text { Taux } \\
\text { butyreux }\end{array}$ & $\begin{array}{l}\text { Taux } \\
\text { azoté }\end{array}$ \\
\hline $\begin{array}{l}\text { Quantité } \\
\text { de lait }\end{array}$ & $\begin{array}{l}\text { Lot expé- } \\
\text { rimental } \\
1 \mathrm{X} \\
2 \mathrm{X} .1 \mathrm{X} \\
2 \mathrm{X}\end{array}$ & $\begin{array}{l}0,92 \\
0,89 \\
0,88\end{array}$ & $\begin{array}{l}0,98 \\
0,98 \\
0,96\end{array}$ & $\begin{array}{r}0,03 \\
0,08 \\
-0,22\end{array}$ & $\begin{array}{r}0,04 \\
0,00 \\
-0,23\end{array}$ \\
\hline $\begin{array}{l}\text { Quantité } \\
\text { de } M G\end{array}$ & $\begin{array}{l}1 X \\
2 X .1 X \\
2 X\end{array}$ & & $\begin{array}{l}0,93 \\
0,89 \\
0,88\end{array}$ & $\begin{array}{l}0,41 \\
0,38 \\
0,24\end{array}$ & $\begin{array}{r}0,17 \\
0,11 \\
-0,10\end{array}$ \\
\hline $\begin{array}{c}\text { Quantité } \\
\text { de MA }\end{array}$ & $\begin{array}{l}1 X \\
2 X .1 X \\
2 X\end{array}$ & & & $\begin{array}{r}0,09 \\
-0,02 \\
-0,15\end{array}$ & $\begin{array}{l}0,21 \\
0,21 \\
0,04\end{array}$ \\
\hline $\begin{array}{c}\text { Taux } \\
\text { butyreux }\end{array}$ & $\begin{array}{l}1 X \\
2 X .1 X \\
2 X\end{array}$ & & & & $\begin{array}{l}0,32 \\
0,29 \\
0,31\end{array}$ \\
\hline
\end{tabular}


Bien que non significativement différents entre traitements, du fait des faibles effectifs, ces coefficients indiquent que la réduction du nombre de traites par jour tend, par le biais de la réduction des quantités produites qu'elle entraîne, à accroître les liaisons phénotypiques entre caractères et notamment à éliminer les relations négatives entre les quantités et les teneurs du lait en ses différents constituants.

\section{4. - Variabilité des performances}

L'examen des variances et coefficients de variation présentés aux tableaux I, 2 et 3 pour différents critères de production laitière indique aussi bien pour les taux que pour les quantités de lait une variabilité phénotypique supérieure des productions enregistrées avec une seule traite journalière. Les coefficients de variation étant en général plus élevés pour le lot IX que pour le lot $2 X$. IX. Ceci traduit probablement les différences de distribution des productions que 1'on peut constater sur la figure 3 , même si les effectifs limités ne permettent pas de les tester.
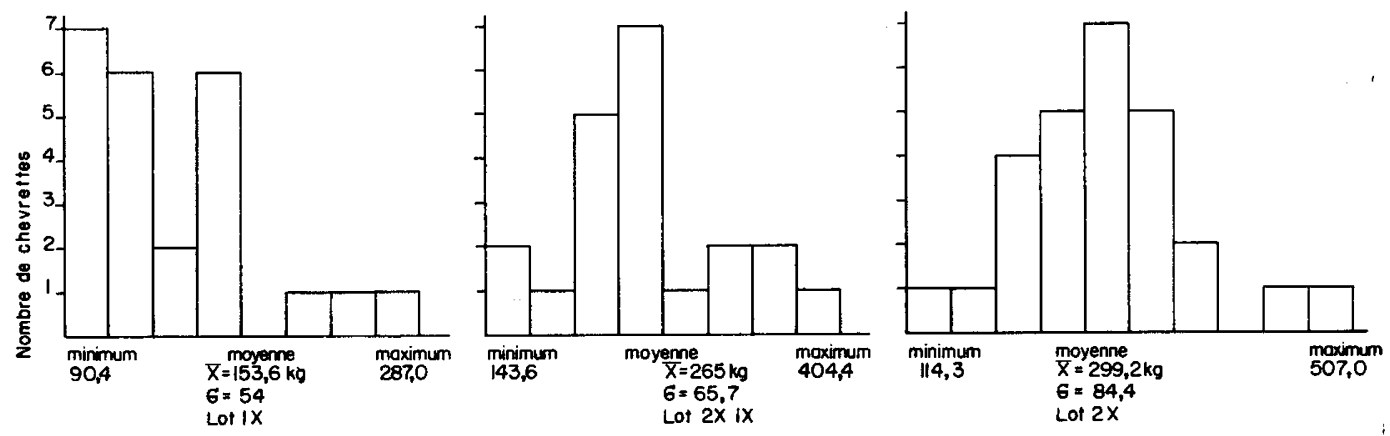

FIG. 3. - Distributions des productions laitières en 110 jours de lactation par classes de demi-écart-type

En effet la distribution des productions de lait en r Io jours de lactation s'écarte notablement, pour le lot IX, de la distribution normale que l'on peut admettre pour le lot $2 \mathrm{X}$. Un nombre relativement important de chevrettes n'a pas été capable de produire plus d'un $\mathrm{kg}$ de lait par jour en une seule traite, alors que les autres en ont produit une quantité variable dans les mêmes conditions. Par ailleurs, la différence de variabilité du lot IX est d'autant plus importante que la lactation est plus avancée, ce qui semblerait traduire des différences individuelles importantes, quant à la persistance de la lactation avec une seule traite par jour. La variance du coefficient de persistance est d'ailleurs supérieure et bien plus grande encore dans le cas des animaux du lot $2 \mathrm{X}$. IX qui ont préalablement été traits deux fois par jour. L'existence de telles différences d'adaptation individuelle à la réduction du nombre de traites est confirmée par la comparaison des coefficients de corrélation observés entre les quantités de lait produites pendant les 54 premiers jours de lactation et les 56 jours suivants. Ces coefficients s'élèvent en effet à 0,66 et 0,77 respectivement pour les lots $\mathrm{IX}$ et $2 \mathrm{X}$ et à 0,53 seulement pour le lot $2 \mathrm{X}$. IX à cause du changement brutal de système en cours de lactation. 


\section{5. - État sanitaire de la mamelle et croissance}

Trois cas de mammite clinique ont été observés pendant 1'expérience à raison d'un animal atteint dans chaque lot. L'examen des résultats de CMT, dont l'efficacité chez la Chèvre reste douteuse compte tenu de la difficulté de les apprécier, ne révèle pas non plus de différences notables entre les lots. On observe par contre des différences très significatives du nombre de leucocytes/ml de lait entre le lot IX $(\simeq$ I IO0 000) et le lot $2 \mathrm{X}(\simeq 300$ 000) ou pour le lot $2 \mathrm{X}$. IX entre le lait produit avant le quatrième contrôle $(\simeq$ I55 00o) et celui produit après ce contrôle $(\simeq 865000)$.

L'examen du tableau 6 où figure l'évolution du poids vif moyen traduit un accroissement plus important du poids des animaux soumis à une seule traite du fait d'une alimentation quasi ad libitum. Malgré le changement de système pour les animaux du lot $2 X$. IX leur poids vif est resté inférieur à celui du lot $2 X$, peut être à cause de leur production plus élevée en début de lactation.

TABLEAU 6

Evolution du poids vif moyen $(\mathrm{kg})$ des chevrettes au cours de leur première lactation

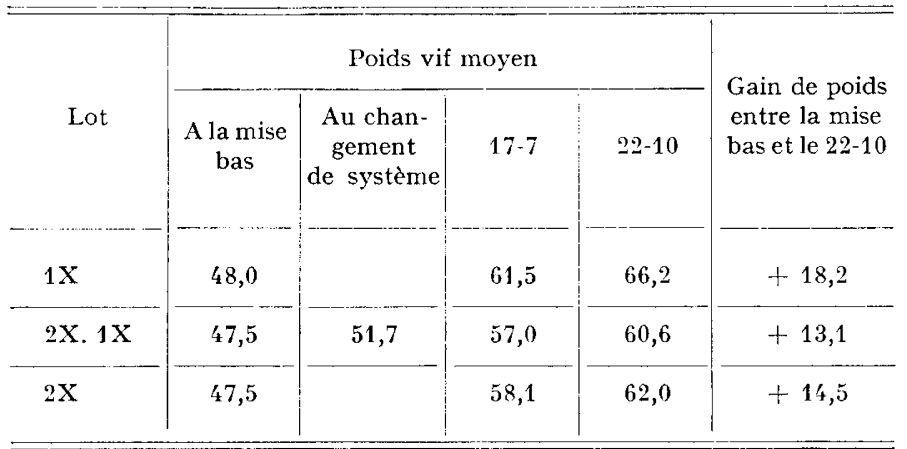

\section{DISCUSSION}

La suppression d'une traite pendant tout ou partie de la lactation entraîne chez la Chèvre des diminutions de production importantes et comparables à celles observées chez les bovins par Woodward (I93I) (56 p. IOo) et par ClaEsson et al. (I959) qui citent une perte moyenne de $50 \mathrm{p}$. Ioo en première lactation, perte moins importante pendant les I40 premiers jours : 45,6 p. IoO, que pendant les I40 jours suivants : 54, I p. Ioo. Les essais de suppression d'une traite en cours de lactation sont plus difficilement comparables, le changement de système ayant été réalisé pour différentes espèces à différents stades de lactation. Craksson observe une diminution de $30 \mathrm{p}$. Ioo puis de $45 \mathrm{p}$. Ioo au cours des deux premières semaines suivant le changement à 240 jours de lactation. Hesseitine et al. (r953) mentionnent dans le même cas de changement à 250 jours une perte moyenne de $38 \mathrm{p}$. Ioo 
sur la production de lait en fin de lactation. CASU et LABUSSIÈrE (I972) observent chez la Brebis Sarde une diminution de 5,2 p. roo seulement des quantités produites en 147 jours et après 30 à 45 jours d'allaitement puis 15 jours à 2 traites, c'est-à-dire après un début de lactation normal de durée comparable à la nôtre (45 à 60 jours). MORAG (I968) observe chez la Brebis une différence de l'ordre de $20 \mathrm{p}$. Ioo entre les productions de demi-mamelles traites une et deux fois par jour, situation dans laquelle il existe une stimulation hormonale indirecte de la secrétion lactée dans la demi-mamelle soumise à une seule traite. Tous ces auteurs constatent également une légère augmentation des teneurs du lait produit, insuffisante pour compenser de façon sensible les pertes observées pour les quantités de lait. Aucune étude ne fait état d'effets défavorables de ces pratiques sur l'état sanitaire de la mamelle.

L'origine de ces pertes n'a pas été étudiée de façon systématique. La capacité limitée de stockage de la mamelle intervient sûrement avec de fortes variations individuelles pour ralentir la sécrétion lactée au moins au-delà d'un délai de 55 heures. L'insuffisance des stimuli nécessaires aux réflexes d'éjection du lait ne semble pas devoir être invoquée dans ce cas. Il est plus probable que l'application d'une seule traite dès la mise bas ne constitue pas dans la plupart des cas une stimulation suffisante pour la décharge des hormones ante-hypophysaires nécessaires au développement du tissu mammaire. Enfin, la suppression définitive d'une traite pendant la lactation peut entraîner soit l'involution rapide d'un certain nombre d'acini soit la réduction de la charge galactopoiétique de l'anté-hypophyse avec probablement dans tous les cas des variations individuelles importantes. Par contre, il ne semble pas y avoir de différences fondamentales de comportement entre les races ou espèces où le stockage du lait est surtout citernal comme la Chèvre ou la Brebis, et celles où il est davantage alvéolaire comme la Vache.

Du point de vue de la sélection, les résultats obtenus dans cette étude ne peuvent conduire qu'à un certain nombre d'hypothèses.

En premier lieu nous pouvons affirmer qu'il sera pratiquement impossible de combler de façon notable par sélection les importantes différences constatées. En effet, la meilleure lactation du lot IX étant approximativement équivalente à la moyenne de production du lot $2 \mathrm{X}$. IX on peut considérer qu'il s'agit là d'une limite. Ce qui ne laisse rien présumer sur l'intérêt d'une telle sélection par comparaison avec une sélection classique à deux traites.

Les résultats obtenus avec une seule traite par jour mettent en évidence une variabilité individuelle supérieure pour un certain nombre de caractères qui ont aussi leur importance dans un système à deux traites, par exemple la durée de lactation, ou la persistance; ou bien encore la capacité de la mamelle ou l'aptitude à développer le tissu mammaire avec un minimum de stimulation. Dans la mesure où la variabilité génétique ainsi que l'héritabilité seraient plus élevées, ou les corrélations génétiques plus favorables dans un tel milieu, on peut penser qu'une sélection indirecte pourrait être dans certains cas plus efficace : par exemple, pour sélectionner sur la persistance et non sur la production maximum, ou bien encore pour améliorer simultanément quantités produites et taux. Enfin, on peut penser qu'un tel milieu de sélection serait plus susceptible de fournir des animaux mieux adaptés à des conditions variables d'exploitation simplifiée pour les techniques de traite, notamment dans la mesure où il semble exister des interactions génotype-milieu de traite. 


\section{CONCLUSION}

En définitive, il semble d'un point de vue pratique que la suppression d'une traite pendant tout ou partie de la lactation entraîne chez les chèvres, de variété Alpine tout au moins, des diminutions importantes de production, comparables à celles observées chez les bovins. Ces pertes rendent impossible une application directe de ces techniques simplifiées de traite. La suppression d'une traite, plus tardivement que dans cet essai, est peut-être intéressante chez la Chèvre dans la mesure où les mises bas étant groupées cette technique peut être appliquée à l'ensemble du troupeau au même moment, et libérer en partie la main d'œuvre pendant les périodes d'été et d'automne.

Sur le plan génétique il est vraisemblable qu'une sélection sur la production en une seule traite conduirait à un progrès notable à court terme, par élimination dans la première phase des animaux non adaptés. A plus long terme il convient de comparer son efficacité à celle d'une sélection classique sur deux traites. Elle pourrait alors ne se justifier que si elle conduit à l'obtention d'animaux également mieux adaptés aux conditions normales actuelles ou à des conditions variables de traite simplifiée envisageables à terme pour des raisons économiques et sociales.

Rę̧u pour publication en décembre 1974.

\section{REMERCIEMENTS}

Les auteurs tiennent à remercier M. J. LABUSSIERE et G. RICORDEAU pour leurs commentaires et suggestions à la lecture du manuscrit.

\section{SUMMARY}

\section{EFFECTS OF DIFFERENT MILKING FREQUENCIES}

ON MILK YIELD IN GOATS

Reducing the number of milkings of dairy females may improve management conditions as long as milk yield is not too seriously affected. Selection of animals adapted to various milking conditions can be studied at less cost for goats. Phenotypic effects of different milking systems on milk yield of young goats in first lactation are analyzed here. 83 crossed females of alpine type (Chamoisée $\times$ Saanen) born in 1972 in our experimental herd are divided into 3 lots with the following treatments : lot $\mathrm{IX}$ : one milking per day; lot $2 \mathrm{X}: 2$ milkings per day; lot $2 \mathrm{X}$. $\mathrm{rX}$ : 2 milkings for 2 months and then only one milking.

The difference in yield between lots $\mathrm{IX}$ and $2 \mathrm{X}$ is maximum and of the order of $50 \mathrm{p}$. roo about the second month of lactation; it then diminishes because yield persists better when there is only one milking. Differences in total overall yield as compared to lot $2 \mathrm{X}$ are $20 \mathrm{p}$. roo and $45 \mathrm{p}$. 100 for lots $2 X$. IX and IX, respectively. Butterfat and Protein contents are slightly higher during $2 / 3$ of the lactation fort lot IX, but for lot $2 X$. IX they are comparable to lot $2 X$, in spite of the change during lactation to one milking. Cutting out one milking during all or part of a lactation seems generally to cause earlier drying off ( $-I_{4}$ days for lot $\mathrm{IX}$ and $-\mathrm{I} 2$ days for lot $2 \mathrm{X}$. $\left.\mathrm{IX}\right)$. Due to lower milk yield, it also tends to increase positive relations and reduce phenotypic oppositions 
between milk quantity and contents. Variability of performances recorded with a single milking per day is higher in terms of coefficient of variation, namely as concerns persistency for lot $2 X$. $\mathbf{I X}$. This seems to express large individual differences in adaptation, which are confirmed by a nonnormal distribution of yields obtained with a single milking. While differences between treatments in relation to the health of the udder are not observed, cutting out one milking causes large increase in the number of leucocytes in the milk.

Selecting animals adapted to one milking may be efficient, at least on a short-term basis, but it is only justified if animals are obtained which are also better adapted to varied conditions of simplified milking.

\section{RÉFÉRENCES BIBLIOGRAPHIQUES}

Casu S., Labussiere J., r972. Premiers résultats concernant la suppression d'une ou plusieurs traites par semaine chez la Brebis Sarde. Ann. Zootech., 21, 223-232.

Claesson O., Hansson A., Gustafson N., Brannang E., r959. Studies on monozygous cattle twins. XVII. Once a day milking compared with twice a day milking. Acta agri. Scand., 9, 38-58.

Claesson O., 1959. Traite une ou deux fois par jour (Suédois). svensk Husdjürsskötsel, 11, 368-385.

Hesseltine W. R., Mochrie R. D., Eaton H. D., Elliott F. I., Beall G., i953. Effects of once daily milking in late lactation. Storrs. Agric. Exp. Sta. Commecticut Bull., 304, p. Io.

LABUSSIERE J., Richard Ph., I965. La traite mécanique. Aspects anatomiques, physiologiques et technologiques. Mise au point bibliographique. Ann. Zootech., 14, 63-126.

Morag M., 1968. The effect of varying the daily milking frequency on the milk yield of the ewe and evidence of the nature of inhibition of milk ejection by half-udder milking. Ann. Zootech., 17, 35I-369.

Woodward T. E., I93I. The production of dairy cows as affected by frequency and regularity of milking and feeding. U.S. Dept. Agric. Cir., r8o. 